\title{
Restriction Enzyme Analysis of Double-Stranded DNA on Pristine Single-Walled Carbon Nanotubes
}

\author{
Shang-Jung Wu, ${ }^{\circledR}$ Nils Schuergers, Kun-Han Lin, Alice J. Gillen, ${ }^{\circledR}$ Clémence Corminboeuf, ${ }^{\circledR}$ \\ and Ardemis A. Boghossian*(1)
}

Institute of Chemical Sciences and Engineering (ISIC), École Polytechnique Fédérale de Lausanne (EPFL), Lausanne CH-1015, Switzerland

Supporting Information

ABSTRACT: Nanoprobes such as single-walled carbon nanotubes (SWCNTs) are capable of label-free detection that benefits from intrinsic and photostable near-infrared fluorescence. Despite the growing number of SWCNT-based applications, uncertainty surrounding the nature of doublestranded DNA (dsDNA) immobilization on pristine SWCNTs has limited their use as optical sensors for probing DNA-protein interactions. To address this limitation, we study enzyme activity on unmodified dsDNA strands immobilized on pristine SWCNTs. Restriction enzyme activity on various dsDNA sequences was used to verify the retention of the dsDNA's native conformation on the nanotube surface and to quantitatively compare the degree of dsDNA accessibility. We report a 2.8 -fold enhancement in initial enzyme activity in the presence of surfactants. Förster resonance electron transfer (FRET) analysis attributes this enhancement to increased dsDNA displacement from the SWCNT surface. Furthermore, the accessibility of native dsDNA was found to vary with DNA configuration and the spacing between the restriction site and the nanotube surface, with a minimum spacing of four base pairs (bp) from the anchoring site needed to preserve enzyme activity. Molecular dynamics (MD) simulations verify that the anchored dsDNA remains within the vicinity of the SWCNT, revealing an unprecedented bimodal displacement of the bp nearest to SWCNT surface. Together, these findings illustrate the successful immobilization of native dsDNA on pristine SWCNTs, offering a new near-infrared platform for exploring vital DNA processes.

KEYWORDS: carbon nanotube, restriction enzyme, DNA conformation, surface functionalization, adsorption

\section{INTRODUCTION}

The discovery of the helical structure of double-stranded DNA (dsDNA) incited technologies that have since shaped modern molecular biology. The omnipresence of DNA in most basic life processes has motivated its incorporation into gene delivery, imaging, and sensing technologies that exploit its biocompatibility, versatile molecular structure, and specificity. ${ }^{1-4}$ When coupled to nanoparticle and fluorescent probes, dsDNA provides a scaffold for studying fundamental processes, such as replication, hybridization, and transcription, as well as bioengineering techniques, such as gene delivery and genome editing. 5,6

Single-walled carbon nanotubes (SWCNTs) are among the most studied nanoparticle probes because of their near-infrared fluorescence, indefinite photostability, and single-molecule detection limits. These beneficial properties are particularly advantageous for both in vivo and in vitro imaging and sensing applications. $^{7-11}$ The noncovalent functionalization of SWCNTs with DNA not only keeps these optoelectronic properties intact, but also increases the solubility and biocompatibility of SWCNTs and bestows biochemical accessibility for further functionalization. ${ }^{12-14}$ In addition,
DNA binding affinity and surface coverage of SWCNTs are length- and nucleotide-specific, ${ }^{15}$ allowing nanotube chirality separation. ${ }^{16}$ The wrapped DNA can further hybridize with complementary sequences or form aptamer structures that bind target molecules with high affinity and specificity, incentivizing its use for optical SWCNT sensing applications. In addition, SWCNTs have been shown to protect DNA from degradation ${ }^{17}$ (see Figure S1 and discussion in the Supporting Information), a critical consideration for gene delivery applications.

Although DNA is the most commonly used polymer for suspending SWCNTs, the majority of SWCNT-based applications rely on noncovalent functionalization with single-stranded DNA (ssDNA) of 20-40 nucleotides (nt). ${ }^{12,13}$ The ssDNA oligonucleotides possess a strong affinity to the SWCNTs that is attributed mainly to $\pi-\pi$ stacking between nucleobases and the SWCNT sidewall. ${ }^{18}$ In contrast, dsDNA has received less attention as it does not efficiently

Received: July 20, 2018

Accepted: October 2, 2018

Published: October 2, 2018 
Table 1. Summary of Previous Proposed Models and Methods

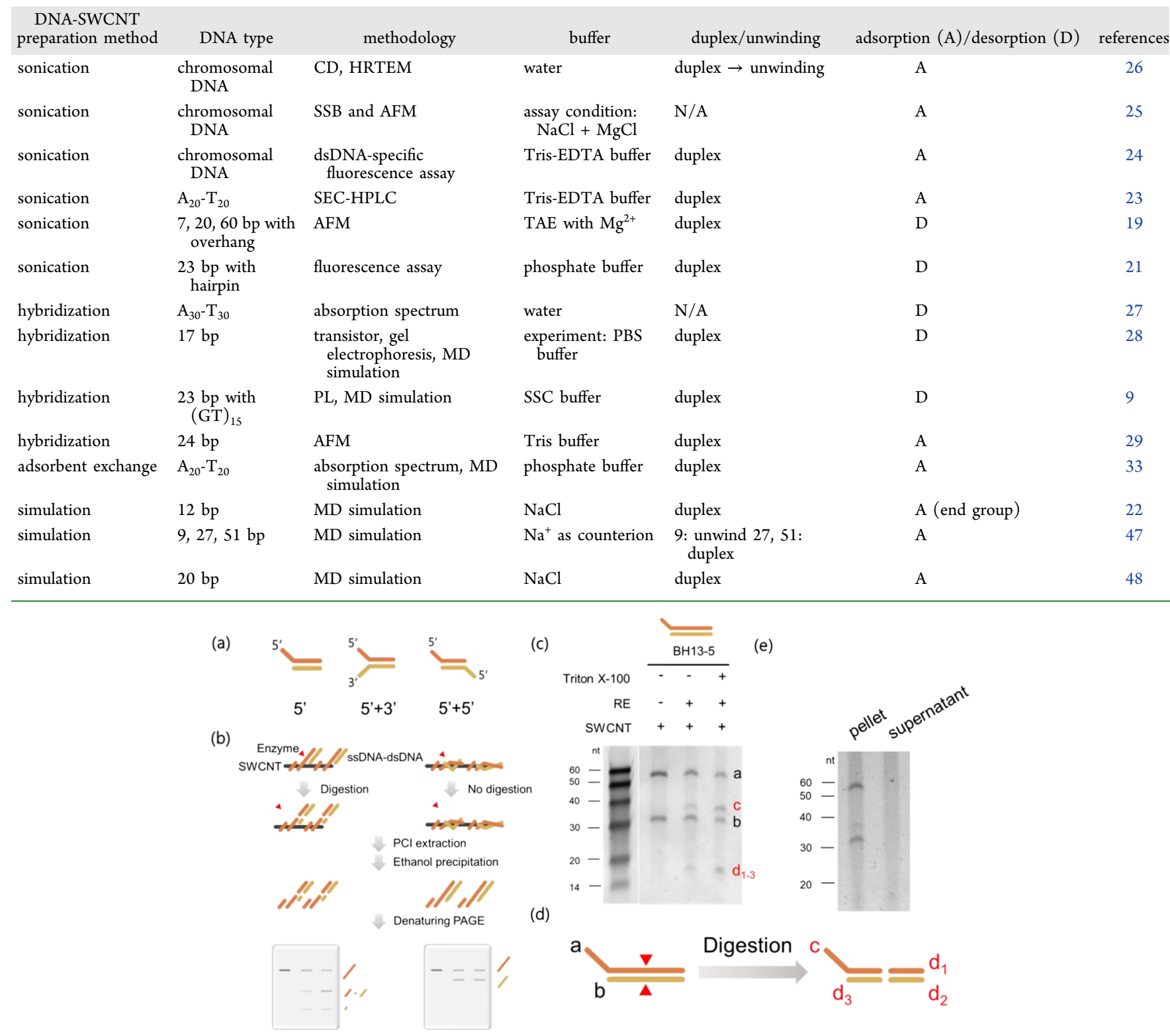

Figure 1. Digestion assay showing dsDNA restriction on the surface of SWCNTs, which is enhanced in the presence of Triton X-100. (a) Schematic of DNA configurations differing in the position of the single-stranded $(\mathrm{GT})_{10}$ overhang(s). (b) Cartoon of the digestion assay. DNASWCNTs were incubated with the corresponding REs whose activities depend on the conformation of the dsDNA on the SWCNT surface. Restriction was followed by phenol-chloroform-isoamyl alcohol extraction and ethanol precipitation to purify DNA from SWCNTs. Recovered DNA fragments were analyzed by denaturing PAGE, and the ratio of different fragments was calculated to determine enzyme activity and dsDNA conformation. (c) BH13-5 DNA extracted from HiPCo SWCNTs was separated on a 12\% denaturing PAGE. Restriction products c (33 nt) and $\mathrm{d}_{1-3}(15 \mathrm{nt})$ were only observed when DNA-SWCNTs were incubated with BamHI. (d) Cartoon showing all possible digestion products. (e) Denaturing PAGE showing DNA recovered from the DNA-SWCNT pellet (i.e., DNA on the SWCNT surface) and from the supernatant (i.e., desorbed DNA) after the digestion reaction.

suspend SWCNTs unless sonicated vigorously. ${ }^{12}$ To circumvent the low binding affinity and functionalize SWCNTs with dsDNA sequences, previous studies have designed hybrid configurations such as dsDNA containing an ssDNA overhang $^{19,20}$ or ssDNA-based hairpin structures. ${ }^{21}$ These studies demonstrated that the hybrids can be employed successfully in optical sensing and nanostructure design applications.

A lack of uniform and precise techniques for dsDNASWCNT characterization has yielded several seemingly conflicting models on the interaction of dsDNA with SWCNTs (Table 1). The weak binding of dsDNA to the SWCNT is believed to emanate from the low affinity between the hydrophilic dsDNA backbone and the hydrophobic SWCNT surface. However, some studies have suggested that van der Waals forces are sufficient to facilitate dsDNA adsorption to the SWCNT surface. ${ }^{22-24}$ Nii et al. demonstrated that ssDNAbinding proteins do not bind to conjugates of chromosomal dsDNA and SWCNTs, while they readily interact with ssDNAfunctionalized SWCNTs, ${ }^{25}$ thereby validating the preservation of the double-stranded structure on SWCNTs. On the other hand, Cathcart et al. investigated SWCNTs suspended with chromosomal DNA from salmon sperm by electron micros- 
Table 2. Short Duplex DNA Oligomers used to Wrap SWCNTs

\begin{tabular}{|c|c|c|c|c|c|}
\hline $\mathrm{DNA}^{a}$ & cutting site ${ }^{b}$ & site-to-end distance ${ }^{c}(\mathrm{bp})$ & dsDNA length (bp) & GC content of dsDNA segment (\%) & overhang configuration $^{d}$ \\
\hline ERV13-5 & EcoRV & 13 & 30 & 47 & $5^{\prime}$ \\
\hline ERV13-53 & EcoRV & 13 & 30 & 47 & $5^{\prime}+3^{\prime}$ \\
\hline ERV13-55 & EcoRV & 13 & 30 & 47 & $5^{\prime}+5^{\prime}$ \\
\hline $\mathrm{BH} 13-5$ & BamHI & 13 & 30 & 53 & $5^{\prime}$ \\
\hline $\mathrm{BH} 13-53$ & BamHI & 13 & 30 & 53 & $5^{\prime}+3^{\prime}$ \\
\hline BH13-55 & BamHI & 13 & 30 & 53 & $5^{\prime}+5^{\prime}$ \\
\hline BHH13-5 & BamHI & 13 & 30 & 70 & $5^{\prime}$ \\
\hline BHL13-5 & BamHI & 13 & 30 & 30 & $5^{\prime}$ \\
\hline BH10-5 & BamHI & 10 & 27 & 52 & $5^{\prime}$ \\
\hline BH7-5 & BamHI & 7 & 24 & 50 & $5^{\prime}$ \\
\hline BH4-5 & BamHI & 4 & 21 & 53 & $5^{\prime}$ \\
\hline $\mathrm{BH} 2-5$ & BamHI & 2 & 19 & 53 & $5^{\prime}$ \\
\hline
\end{tabular}

${ }^{a}$ DNA sequences are named based on the RE cutting site, site-to-end distance, and configuration, consecutively. ${ }^{b}$ The 6 -bp recognition site for BamHI (5'-GGATCC-3') or EcoRV (5'-GATATC- $\left.3^{\prime}\right)$ is included in the dsDNA sequence. ${ }^{c}$ The site-to-end distance is defined as the length between cutting site and the closest overhanging end. ${ }^{d}$ The overhang configuration is defined as the position(s) where a $(\mathrm{GT})_{10}$ overhang is added to one or both complementary strands.

copy and circular dichroism analysis. ${ }^{26}$ They concluded that following sonication, nanotubes are initially stabilized by dangling ssDNA ends from which the dsDNA slowly denatures to form an ordered ssDNA coating around the SWCNT within 20-50 days.

Similar disparities have been reported for the hybridization of complementary sequences to ssDNA on the surface of SWCNTs. ${ }^{9,27-29}$ For example, on the basis of electrophoresis and molecular dynamics (MD) simulation results, Jung et al. suggested that 17 base pairs (bp) dsDNA duplexes formed by hybridization desorb from the nanotube surface. ${ }^{28}$ On the other hand, Jeng et al. used atomic force microscopy (AFM) imaging to show that a random $24 \mathrm{bp}$ dsDNA will remain adsorbed on a nanotube surface after duplex formation. ${ }^{29}$ However, AFM imaging did not allow the authors to distinguish whether the dsDNA duplex adopts a canonical Btype conformation or some nonnatural conformation on the SWCNT surface.

Differences in sequences, observed time scales, varying degrees of charge screening by counter ions, and other factors in these studies may contribute to the observed variation in the dsDNA-SWCNT behavior. The field thus currently lacks a fundamental understanding of the nature of the dsDNASWCNT interaction, the preservation of the dsDNA structure, and its accessibility while wrapped on SWCNTs. In this work, we study the structure of dsDNA strands on SWCNTs by analyzing the interaction of dsDNA-modifying type-II restriction enzymes (REs) with DNA-SWCNT hybrids. Type-II REs recognize short palindromic dsDNA sequences and cleave the phosphodiester bond of the DNA backbone within their respective recognition sites. ${ }^{30}$ Type-II REmediated DNA cleavage is widely used in different techniques, such as restriction fragment length polymorphism, single nucleotide polymorphism, and post-modification analysis as the cleavage activity can be easily investigated by gel electrophoresis. Importantly, these enzymes are mostly specific for dsDNA in the native B-DNA conformation. ${ }^{31,32}$ We take advantage of this conformational specificity to understand the structure and stability of DNA duplexes in the vicinity of the SWCNT surface. Additional Förster resonance electron transfer (FRET) measurements and $\mathrm{MD}$ simulation results show that short dsDNA strands can retain their native conformation in the proximity of a SWCNT surface.

\section{RESULTS}

The enzymatic digestion assay developed in this study (Figure 1) allows us to quantify the amount of accessible native dsDNA on the nanotube surface. HiPCo SWCNTs were functionalized with short $(\leq 30 \mathrm{bp})$ dsDNA sequences containing an endonuclease recognition site and a singlestranded $(\mathrm{GT})_{10}$ overhang on either one or both strands (Figure 1a, Table 2; see Table S1 for exact sequences). DNASWCNT hybrids were prepared by a sodium cholate (SC)exchange method adopted from Shiraki et al., ${ }^{33}$ as this method minimizes SWCNT damage and increases yields of native dsDNA on SWCNTs (see conclusion and discussion in Supporting Information for a comparison of DNA-SWCNT hybrids prepared by different methods). Free DNA was thoroughly removed (Figure S2), and the suspension (around $1 \mathrm{mg} / \mathrm{L}$ of SWCNT) was incubated with the BamHI restriction enzyme to cleave native dsDNA into smaller fragments. Total DNA was subsequently purified from the SWCNT suspension by phenol-chloroform-isoamyl alcohol extraction (Figure S3), concentrated, and analyzed by denaturing gel-electrophoresis (Figure 1b). Because BamHI is specific to DNA in the B-conformation, ${ }^{31}$ the enzyme activity is directly related to the amount of accessible DNA in its native configuration.

This protocol was first applied to SWCNTs wrapped with dsDNA containing a single 5 -overhang and a GC content of 53\% (BH13-5). Enzymatic digestion (Figure 1c) leads to the appearance of two extra bands around 33 and $15 \mathrm{nt}$, which correspond to the lengths of the expected fragments $c$ and $\mathbf{d}_{1-3}$, as shown in Figure $1 \mathrm{~d}$. These bands resulted from the specific restriction at the single BamHI site of sequence BH135 , as no cutting was observed for single-stranded sequences or a hybrid sequence without a BamHI recognition site (Figure S4). In contrast, the uncut bands shown as fragments $\mathbf{a}$ and $\mathbf{b}$ may represent uncut dsDNA as well as any adsorbed ssDNA strands that may have remained following annealing. To eliminate the possibility that free oligonucleotides detaching from the SWCNTs contribute to the observed cutting, we independently analyzed DNA fragments extracted from the SWCNT-containing pellet and the supernatant of an ultrafiltrated solution directly following enzymatic restriction (Figure 1e). Fragments a, b, and $\mathbf{c}$ were only found in the pellet fraction containing the SWCNTs, and no desorbed DNA 
(a)

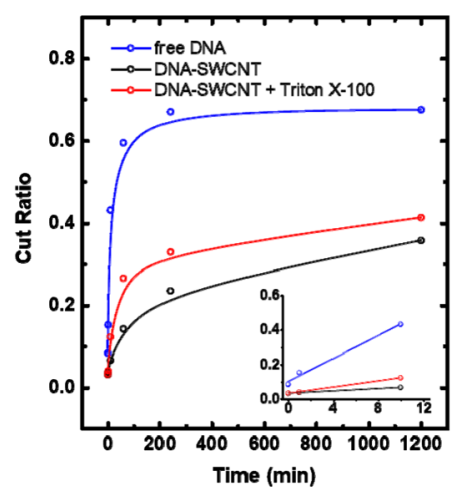

(b)

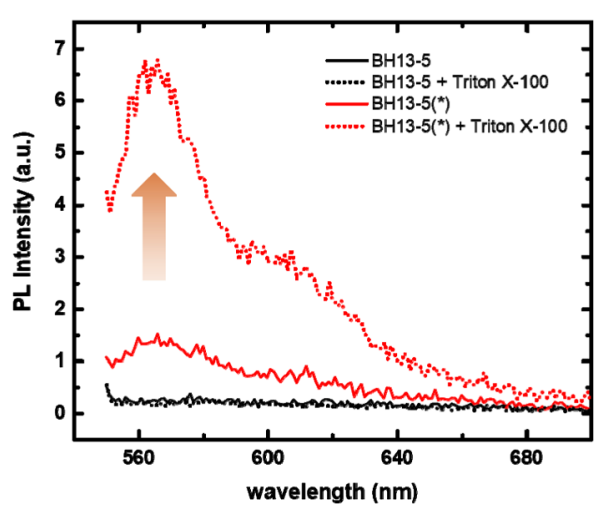

(c)

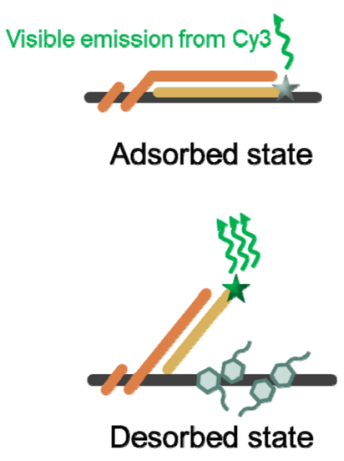

Figure 2. Triton X-100 enhances BamHI activity by increasing the mean distance between dsDNA and SWCNT. (a) BamHI digestion kinetics for free DNA, DNA-SWCNTs, and DNA-SWCNTs with Triton X-100. The inset shows the initial slopes fitted by linear regression. (b) FRET assay to monitor the dsDNA-SWCNT distance. DNA-SWCNT hybrids of BH13-5 or BH13-5(*), which has a Cy3 fluorophore attached at the opposite end of the (GT) $)_{10}$ overhang, were excited at $532 \mathrm{~nm}$. Emission spectra are shown before (solid lines) and after (dash lines) the addition of 0.002 wt \% Triton X-100. (c) Cartoon depiction of adsorbed and desorbed states of DNA on the SWCNT surface. Triton X-100 (hexagons) stabilizes the SWCNT surface that is exposed to water, allowing the dsDNA fragment to desorb from the SWCNT surface.

was detected in the supernatant, confirming that restriction occurred at the SWCNT surface. We noted that despite the observed surface-bound activity, dsDNA restriction was not complete, and enzyme activity was notably decreased when compared to the restriction rate of free DNA (Figure S5).

In order to quantify the number of cutting events, we examined the relative enzyme activity $\left(A_{\text {rel }}\right)$. We define this variable as the cut ratio (CR) of DNA in the SWCNT hybrid normalized to the CR of free DNA with the same sequence.

$$
A_{\text {rel }}=\frac{\mathrm{CR}_{\mathrm{DNA}-\mathrm{SWCNT}}}{\mathrm{CR}_{\text {free DNA }}}
$$

This normalization therefore accounts for contributions from any unwanted ssDNA which may still be present during the digestion assay. The $\mathrm{CR}$ was calculated from the fluorescence intensity observed for bands $\mathbf{a}$ and $\mathbf{c}$ in the PAGE gel ( $I_{\mathrm{a}}$ and $I_{\mathrm{c}}$ ) normalized with respect to the corresponding DNA length $\left(L_{\mathrm{a}}\right.$ and $\left.L_{\mathrm{c}}\right)$, assuming that fluorescence intensity is proportional to DNA length.

$$
\mathrm{CR}=\frac{I_{\mathrm{c}} / L_{\mathrm{c}}}{I_{\mathrm{a}} / L_{\mathrm{a}}+I_{\mathrm{c}} / L_{\mathrm{c}}}
$$

The relative activity of BamHI on BH13-5 SWCNT hybrids was calculated to be $A_{\text {rel }}=0.26$, indicating a strong negative impact on enzymatic DNA cleavage due to the presence of SWCNTs.

Cleavage of DNA by type-II REs consists of three general mechanistic steps: first, the enzyme nonspecifically binds to DNA; second, it slides or hops along the DNA helix until it encounters a specific recognition site; and third, the enzyme cleaves the phosphate-sugar backbone and diffuses from the DNA. Reduced enzyme activity on DNA-SWCNT hybrids is likely caused by either (i) competing adsorption of the enzyme on the SWCNT surface or (ii) hindered DNA accessibility for the enzyme due to the presence of the SWCNT. We explored enzyme adsorption (case (i)) by mixing free DNA and BamHI in the presence of SWCNTs suspended in $0.02 \mathrm{wt} \%$ SC. Although the SC concentration was deliberately chosen to not fully cover the SWCNT ${ }^{34}$ and allow BamHI to adsorb on the surface, the presence of SWCNTs in the reaction mix did not significantly influence the yield of cleavage products as $96 \%$
BamHI activity was retained (Figure S6). This finding indicates that enzyme adsorption is not limiting DNA cleavage.

Next, we investigated if close proximity of the dsDNA to the SWCNT surface leads to steric hindrance or induces conformational changes on the DNA-helix (case (ii)). The accessibility of the dsDNA was studied by tuning the strength of the dsDNA-SWCNT interaction through surfactant addition. This approach is based on work by Harvey et al., ${ }^{9}$ who showed that certain surfactants can be used to weaken the interaction of DNA-RNA duplexes with the SWCNT surface. Our measurements showed that the addition of 0.002 wt \% Triton X-100, a nonionic surfactant that does not denature proteins at low concentrations, ${ }^{35}$ leads to an $8 \mathrm{~nm}$ blue shift of the DNA-SWCNT photoluminescence (Figure S7). This blue-shifting was attributed to altered dsDNA binding to the SWCNT without desorbing ssDNA from the surface. ${ }^{9}$ In addition, the presence of Triton $\mathrm{X}-100$ in the BH13-5SWCNT digestion assay boosted the relative activity of the BamHI digestion by 2.3-fold to 0.60 compared to the case without surfactant (Figure 1c).

The influence of Triton X-100 was further studied by measuring the reaction kinetics of DNA cleavage (Figure 2a). The initial linear rate of DNA cleavage $\left(r_{\mathrm{i}}\right)$ in DNA-SWCNT hybrids is $0.0034 \mathrm{~min}^{-1}$, which is 10 times lower than the $r_{\mathrm{i}}$ for free DNA $\left(0.033 \mathrm{~min}^{-1}\right)$. By introducing Triton X-100 to the system, $r_{\mathrm{i}}$ was increased 2.8 -fold $\left(0.0091 \mathrm{~min}^{-1}\right)$, implying that enzyme kinetics were negatively affected by reduced DNA accessibility on the SWCNT surface. The addition of Triton X100 can thus enhance DNA accessibility and facilitate binding and/or cutting by BamHI.

On the basis of these findings, we hypothesize that the amphiphilic Triton X-100 destabilizes the dsDNA-SWCNT interaction by preferentially binding to the hydrophobic SWCNT surface. Consequently, native dsDNA is displaced from the nanotube surface to promote accessibility by BamHI. To verify dsDNA displacement by Triton X-100, we employed FRET analysis. FRET has previously been used to study ssDNA and/or RNA hybridization events with fluorophorelabeled ssDNA-SWCNTs. ${ }^{9,36}$ The enhanced fluorescence intensity on the addition of complementary sequences was attributed to an increased mean distance between the dye and 
(a)

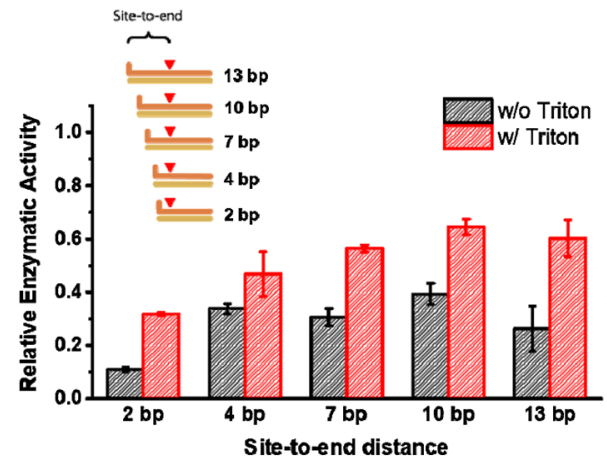

(b)

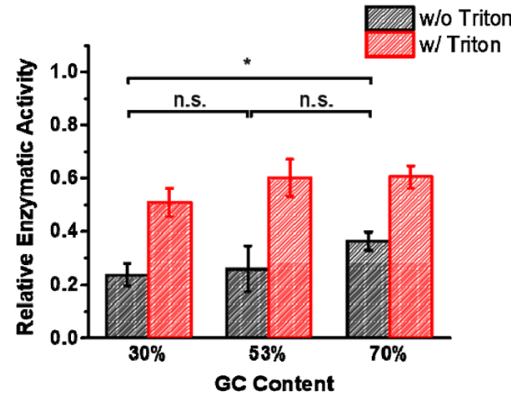

(c)

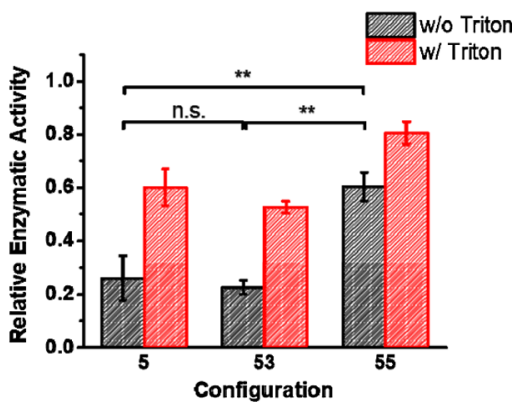

Figure 3. RE activity depends on the sequence and configuration of DNA wrapped on SWCNTs. Fragment analysis by denaturing PAGE was used to quantify the relative enzyme activity, as defined in eq 2 and plotted against (a) site-to-end distance, (b) GC content of the dsDNA sequence, and (c) configuration of $(\mathrm{GT})_{10}$ overhang. All results are mean values of independent triplicates, with the error bar representing 1 S.D. Significance was evaluated by a two-sample $t$-test $\left(*=p<0.05,{ }^{*}=p<0.01\right)$.

the SWCNT surface. Here, we functionalized SWCNTs with DNA containing an overhang in the $\mathbf{5}$ configuration and a Cy3 fluorophore attached to the opposite 5 '-end of the complementary strand (BH13-5(*); Table S1). We excited SWCNT-BH13-5 $(*)$ conjugates at $532 \mathrm{~nm}$ and collected visible-range fluorescence spectra to measure changes in energy transfer. While Triton X-100 had a minimal effect on Cy3 fluorescence for free BH13-5(*) (Figure S8), it yielded a 6-fold fluorescence increase when the DNA was conjugated to the SWCNT (Figure 2b). Because FRET efficiency $(E)$ is inversely related to the distance according to the equation

$$
E=\frac{1}{1+\left(r / R_{0}\right)^{6}}
$$

where $r$ is the distance between the emitter and the quencher and $R_{0}$ is the Förster distance, the 6-fold decrease in efficiency in the presence of surfactant suggests an increase in the mean distance between the 5'-Cy3 and the SWCNT. Assuming the change of FRET efficiency is proportional to the change of fluorescence, $r \approx 9.9 \mathrm{~nm}$ (the length of the dsDNA) in the presence of surfactants, and $r \approx 0.8 \mathrm{~nm}$ in the absence of surfactants (i.e., hydrodynamic radius of $\mathrm{Cy} 3$ ), ${ }^{37}$ we predicted the $R_{0}$ of Cy3 and SWCNT is on the order of $\sim 8 \mathrm{~nm}$. This value falls within the range of previously reported values of $R_{0}$ of Cy3 coupled with a nanoparticle. ${ }^{38}$ The close agreement between the predicted and reported values of $R_{0}$ thus confirms that the ssDNA anchor remains attached, while the dsDNA strand is displaced.

The effects of the DNA sequence and configuration on enzyme activity were also studied. Specifically, we varied the recognition site-to-end distance, GC content, and the configuration of the $(\mathrm{GT})_{10}$ overhang(s), as summarized in Figure 3. First, we tested five different variants with restriction site-to-end spacings varying from 2 to $13 \mathrm{bp}$. In the absence of
Triton X-100, the relative activity of BamHI was significantly decreased only when the recognition site was within $2 \mathrm{bp}$ from the anchoring $(\mathrm{GT})_{10}$ overhang (i.e., BH2-5) (Figure 3a), whereas placing the cut site more than $4 \mathrm{bp}$ from the overhang (i.e., BH4-5 to BH13-5) leads to a 3-4-fold increase in relative activity. This finding shows that the SWCNT has a substantial negative impact on enzyme activity when the recognition site is in close proximity to the wrapping anchor. This observation is in agreement with the results of Shiraki et al., ${ }^{33}$ who predicted that dsDNA termini can be partially disrupted by the shortlived exchange of favorable base pairings with $\pi-\pi$ interactions along the SWCNT surface, a phenomenon that has been described as "fraying" in another study. ${ }^{26}$ As our results imply that this loss of the native conformation is restricted to the first few bp near the ssDNA anchor, we find that positioning sequence elements at least $4 \mathrm{bp}$ into the dsDNA region is crucial for optimizing applications relying on protein-dsDNA interactions. Similarly, Landry et al. previously confirmed that a spacer of at least six units of ethyleneglycol between the anchoring DNA and the aptamer is necessary to achieve protein-aptamer binding on an SWCNT surface. ${ }^{39}$

The addition of Triton X-100 increased BamHI activity for all restriction site-to-end distances tested. The effect was especially pronounced in the case of $\mathrm{BH} 2-5$ (site-to-end distance of 2 bp, as shown in Figure 3a) where BamHI showed a 3-fold increase in activity in the presence of surfactants. This corroborates the idea that Triton X-100, by shielding the SWCNT surface, weakens dsDNA-nanotube interactions and allows dsDNA to move away from the SWCNT. Conversely, in the absence of surfactants, the increased steric hindrance of the SWCNT is shown to disrupt enzyme activity. Moreover, a strong positive correlation between the site-to-end distance and relative enzyme activity was observed (Spearman correlation $=0.81$, with $p<0.001)$. As in the case of 
(a) ECORV (1RVB) BamHI (1ESG)

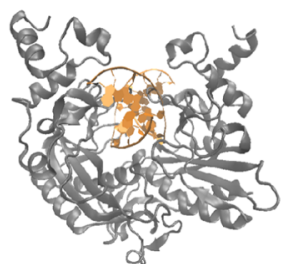

(b)

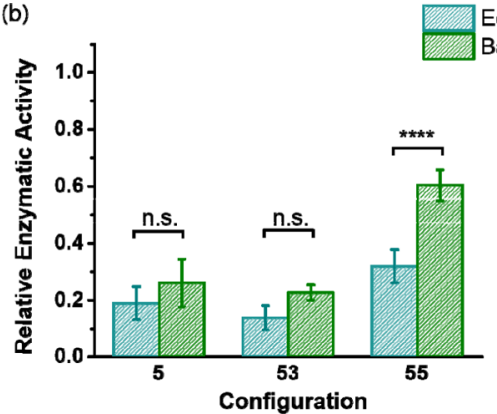

Figure 4. Different enzymes exhibit distinct restriction kinetics toward dsDNA of varying overhang configurations. (a) Crystal structure of EcoRV (PDB: 1RVB) ${ }^{42}$ and BamHI (PDB: 1ESG) ${ }^{45}$ binding to DNA (orange). (b) Comparison of EcoRV and BamHI activity on dsDNA-SWCNT with different $(\mathrm{GT})_{10}$ overhang configurations. Denaturing PAGE fragment analysis was used to quantify RE activity. Significance was evaluated by a two-sample $t$-test $(* * * *=p<0.0001)$.
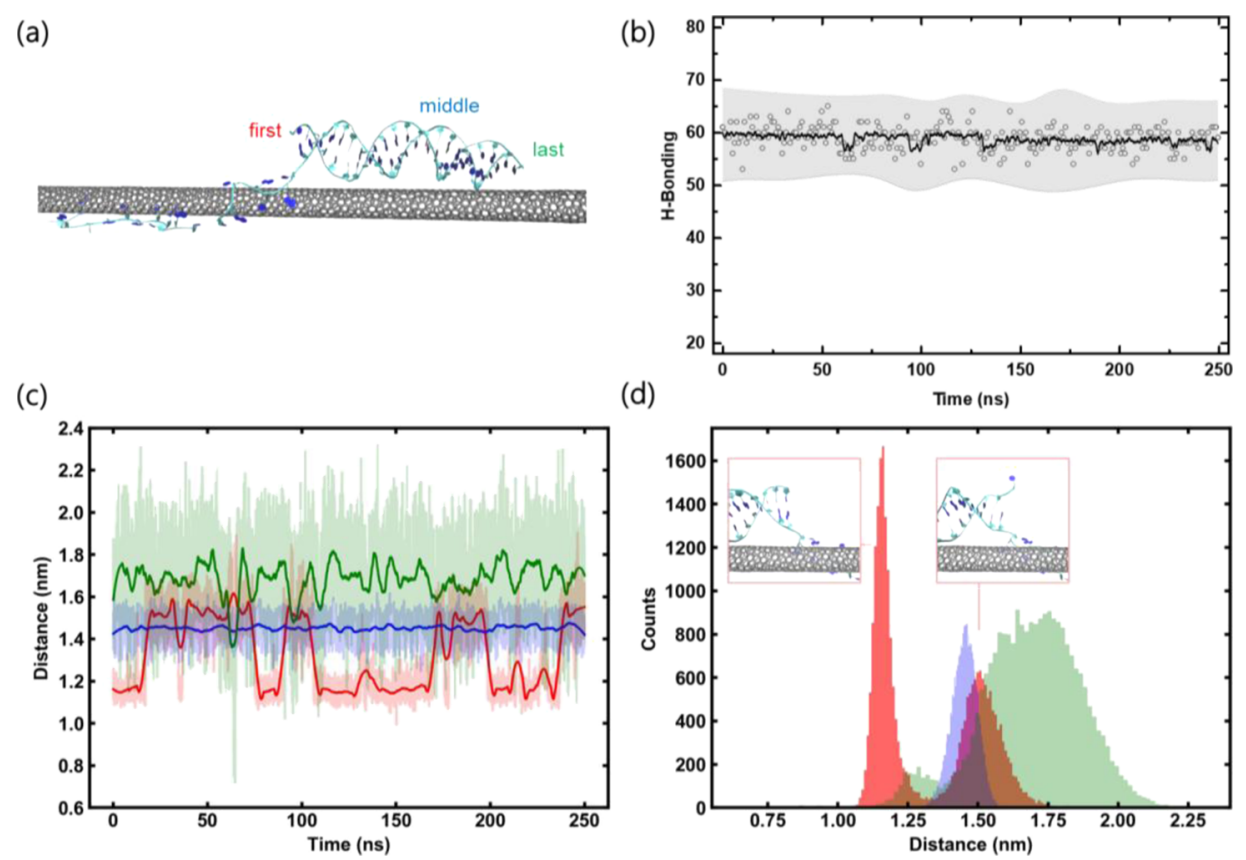

Figure 5. MD simulation of a DNA-SWCNT complex predicts stable dsDNA pairing while adsorbed on an SWCNT surface. (a) Final structure after $250 \mathrm{~ns}$ production run showing the dsDNA aligned along the SWCNT. (b) Number of hydrogen bonds formed over time calculated with a threshold of $0.35 \mathrm{~nm}$ and $30^{\circ}$. (c) Distance between the SWCNT surface and the center of mass for the first ( $3^{\prime}$ end of antisense strand, in red), middle (blue), and the last ( $5^{\prime}$ end of antisense strand, in green) bp shows minimal variance over time. (d) Histogram of the bp-SWCNT distance. The insets depict snapshots of the first bp in the far (at $d=1.5 \mathrm{~nm}$ ) state and the near (at $d=1.1 \mathrm{~nm}$ ) state, showing the temporary disruption of the Watson-Crick pairing in the far state.

surfactant addition, the increased distance from the SWCNT surface is believed to promote enzyme activity through increased dsDNA accessibility.

The stability of dsDNA can be improved by increasing the number of GC pairs, which form an additional third hydrogen bond and have a more favorable base stacking energy compared to AT pairs. Nonetheless, GC content showed only a minor influence on relative BamHI activity when comparing SWCNTs wrapped with $30 \%$ GC dsDNA sequences (BHL13-5) to 70\% GC sequences (BHH13-5) (Figure $3 \mathrm{~b}$ ). The addition of Triton X-100 increased BamHI activity for all GC variants tested to an extent that negated the small differences seen in the absence of the surfactant. Independent of their GC content, the $30 \mathrm{bp}$ DNA duplexes used in this study are sufficiently stable to prevent DNA dehybridization in favor of competitive SWCNT interactions.
Accordingly, dsDNA stability is unlikely to be the limiting factor for BamHI activity.

In contrast to GC content, the configuration of the tested DNA hybrids had a strong impact on enzyme activity (Figure $3 \mathrm{c}$ ). While there was little difference in relative enzyme activity between DNA with a single (BH13-5) or double (BH13-53) overhang at the same terminus, activity increased markedly when two overhangs were added at different DNA termini (BH13-55). The two overhangs are capable of either binding on the surface of the same nanotube or the surfaces of two distinct nanotubes. Han et al. showed that the majority of SWCNTs suspended with DNA containing two overhangs in a similar 55 configuration assume the latter arrangement, allowing dsDNA bridging between two distinct nanotubes. ${ }^{19}$ Such bridging would introduce an additional tensile strain on the dsDNA strands that could displace them from the nanotube surface and facilitate dsDNA cleavage by BamHI. 
Although wrapping of the BH13-55 on the same nanotube could negatively impact enzyme activity, our results indicate that this effect could be outweighed by the majority of nanotubes that are expected to show a relative increase from the dsDNA bridging. Again, relative activity of all configurations was promoted by the addition of Triton X-100.

Furthermore, we compared the activity of different digestion enzymes on dsDNA-SWCNT hybrids (Figure 4a). BamHI and EcoRV are REs with comparable DNA-binding constants (EcoRV: $10.0 \mu \mathrm{M}$ and BamHI: $11.0 \mu \mathrm{M}$ for nonspecific DNA). ${ }^{40}$ The relative activity of EcoRV was similar to BamHI for dsDNA-SWCNTs with duplexes in the $\mathbf{5}$ and $\mathbf{5 3}$ configurations (Figure 4b). As with BamHI, EcoRV showed enhanced activity with duplexes in the $\mathbf{5 5}$ configuration compared to the $\mathbf{5}$ and $\mathbf{5 3}$ configurations, in agreement with the hypothesis that the favored bridging conformation may contribute to increased DNA accessibility. However, this enhancement was less pronounced than that observed with BamHI, and we consequently observed a significant difference between the enzymes' activities with this dsDNA configuration $(p<0.0001)$. A comparison of the crystal structures of the enzymes (BamHI: 1ESG; EcoRV: 1RVB) bound to noncognate DNA shows that EcoRV occupies a larger interaction surface area $\left(2448 \AA^{2}\right)$ than BamHI $\left(631 \AA^{2}\right)\left(\right.$ PDBePISA $\left.^{41}\right)$, though both enzymes are smaller than the ca. $10 \mathrm{~nm}$ distance that is predicted between the bridged nanotubes. ${ }^{19}$ Moreover, upon binding to its recognition sequence, EcoRV needs to bend the DNA in a $50^{\circ} \operatorname{kink}^{42,43}$ to correctly position the phosphodiester bonds for DNA cleavage. This bending could be hampered more by the torsional strain from the DNA bridge compared to BamHI. ${ }^{44}$ Consequently, this torsional strain, combined with the increased interaction surface, may therefore account for the diminished EcoRV enhancement compared to BamHI.

Lastly, we used classical MD simulations (see the Supporting Information for details) to understand the stability and conformation of different regions of dsDNA (BH7-5) on the SWCNT surface (Figure 5a). Ghosh and co-workers have shown that inevitable double-stranded oligonucleotide unzipping is affected by factors such as type of oligonucleotide, salt concentration, and temperature. ${ }^{46}$ Greater base-pair stability has been reported for dsDNA compared to other oligonucleotides, such as siRNA, and for longer sequence lengths. ${ }^{22,47}$ For the experimental conditions used in our study, we observe that dsDNA remains hybridized. In line with this, we did not observe unwinding of the $24 \mathrm{bp}$ duplex over the time scale used in our simulation (Supporting Information video S1). This stability was attributed to the stable canonical WatsonCrick base pairs in the simulated sequence (Figure 5b). The duplex region was shown to largely retain the theoretical 60 hydrogen bonds throughout the simulation for a hydrogen bond pairing cutoff of $0.35 \mathrm{~nm}$ and $30^{\circ} .47$ The observed stability of the DNA duplex along the SWCNT is in agreement with previously published MD simulations for DNA sequences $>12$ bp. $9,22,47,48$

A comparison of the distance $(d)$ between the SWCNT surface and the center of mass of each of the bp of the dsDNA over time showed that the bp closest to the ssDNA anchoring point conveys bimodal displacement with $d$ oscillating between 1.1 and $1.5 \mathrm{~nm}$ (Figure 5c). In the state where the bp was close to the SWCNT, the nucleobases formed canonical H-bonds (inset of Figure 5d). However, as the first bp has lower stability because of less neighboring bases, ${ }^{49}$ the Watson-Crick pairing can be temporally broken, and the fluctuating residue leads to an increase in $d$. This binding disruption is in agreement with the "fraying" phenomenon previously reported in the literature 26,33 and in the discussion above for cut sites within $2 \mathrm{bp}$ of the nanotube surface. In contrast, the central and last bp of the dsDNA segment remained in a relatively constant position with $d=1.5 \pm 0.05$ and $1.7 \pm 0.2 \mathrm{~nm}$, respectively. Although the large standard deviation of $d$ implies that the last bp has the highest tendency to desorb from the SWCNT surface, it stayed within $2.2 \mathrm{~nm}$ throughout the simulation. As all MD simulations previously mentioned neglected the polarization contribution (i.e., a nonpolarizable force field was used, see the Supporting Information), the attractive force between dsDNA and SWCNT is typically underestimated. ${ }^{22}$ Therefore, the adsorption of dsDNA on SWCNTs is likely even more favorable than predicted.

These observations can be reconciled with the observations of Jung et al., ${ }^{28}$ who suggested that the energy released by a 17 bp duplex formation is enough to facilitate desorption of the dsDNA. The values estimated by the authors predict that the $24 \mathrm{bp}$ dsDNA used in our simulation would have a desorption energy of around $56 \mathrm{kcal} / \mathrm{mol}$, which is 2 orders of magnitude larger than thermal fluctuation at ambient conditions. In addition, Harvey et al. also reported adsorption of an unanchored $23 \mathrm{bp}$ dsDNA along the surface of the SWCNT. ' Accordingly, these findings suggest that prehybridized dsDNA remains within the vicinity of the SWCNT surface under ambient conditions. This observation corroborates the results in Figure 3a, which show comparable enzyme activity in the absence of surfactants for cut sites between 2 and $13 \mathrm{bp}$ from the anchoring $(\mathrm{GT})_{10}$, suggesting that the loci of the cut sites are all close to the SWCNT surface.

\section{CONCLUSIONS}

This study presents a systematic investigation of enzyme activity on unmodified DNA strands wrapped on pristine SWCNTs. Whereas previous work on SWCNT-based DNAprotein interactions has focused on the binding activity of proteins to aptamers ${ }^{39}$ or on single-strand DNA-binding proteins, ${ }^{25}$ our findings uniquely explore the effects of enzyme activity, specifically the cutting of the phosphodiester backbone of dsDNA on the SWCNT surface. To the best of our knowledge, this study offers the first experimental verification of a native dsDNA conformation on an SWCNT surface by DNA-protein interaction. Our results show that the DNAprotein interaction strongly depends on DNA accessibility, which can be enhanced through the addition of nonionic surfactants. FRET analysis shows that the surfactant is able to partially displace the dsDNA by diminishing DNA-SWCNT affinity. In addition to DNA-SWCNT affinity, variation in the activities of different enzymes suggests that geometric constraints based on the interaction area and enzyme mechanism may also affect DNA accessibility. Although the precise mode of action of REs on SWCNT-adsorbed dsDNA has not yet been studied, previous studies have explored enzyme behavior in the presence of a competitive DNA-bound protein. Jeltsch et al. showed that REs helically scan along DNA and pause when encountering a bound ligand. ${ }^{50} \mathrm{We}$ propose a similar mechanism for the dsDNA-SWCNT system, where the enzyme helically scans along the dsDNA until it encounters the SWCNT, blocking enzyme sliding and thereby slowing binding-site recognition. 
Alternative models on the interaction of dsDNA with SWCNTs have been reported in the literature (Table 1). Differences in the predicted behaviors can be attributed to the range of experimental conditions, including varying buffer systems and component concentrations that are expected to modulate the dsDNA-SWCNT interaction markedly. Our restriction enzyme digestion assay provides a universal method applicable under varying conditions to evaluate the conformation of dsDNA interacting with SWCNTs. For example, we applied the assay to compare enzyme activity on dsDNASWCNT hybrids prepared using different methods (Figures S9 and S10, and discussion in the Supporting Information). While the assay confirmed that all the tested methods generate native and accessible dsDNA, the yield varied considerably, with the SC-exchange method adopted in this study showing the highest yields. We also applied the assay to monitor the longterm stability of dsDNA in the nanotube hybrid (Figure S11), and we observed negligible denaturation of the dsDNA within 3 months at $4{ }^{\circ} \mathrm{C}$. In addition to its applicability in different conditions, this technique is insensitive to GC content, offering great versatility for studying dsDNA-SWCNT hybrids and applications over a wide range of sequence compositions.

While previous efforts to explore and enhance the interaction between SWCNTs and dsDNA have relied on carboxylated-SWCNTs ${ }^{51}$ or pyrene-modified biomolecules, $^{52,53}$ these approaches show limited application for high-throughput screening or optical sensing. The added flexibility in using pristine SWCNTs, which are specifically capable of autofluorescence, offers a promising prospect for the label-free optical detection of protein-dsDNA interactions. Future applications of this platform therefore include not only exploring other DNA-binding proteins, such as transferases and topoisomerases, but also exploiting the preserved nIR SWCNT fluorescence to optically detect a broad range of enzymatic DNA-protein interactions in vitro and/or in vivo. Such in vivo applications can exploit the temperate properties of Triton X-100, which has been shown to increase nanoparticle uptake and permeabilize cell membranes at low concentrations without curtailing cell viability. ${ }^{54,55}$ Alternatively, the system can benefit from the natural salt concentration gradient that exists across the outer cell membrane to trigger DNA release for gene delivery applications, as described by Ghosh et al. ${ }^{46}$ Thus, by carefully choosing an appropriate DNA configuration, suspension method, and reagent conditions for tuning dsDNA-SWCNT interactions, this platform can enable new dsDNA optical measurements for both fundamental and applied studies.

\section{METHODS AND EXPERIMENTS}

Materials. Purified SWCNTs were purchased from NanoIntegris (HiPCo, batch HP26-019 and HP29-064). BamHI-HF (E163A/ E167T), EcoRV-HF (D19A/E27A), and reaction buffer NEBuffer 4 ( $50 \mathrm{mM}$ potassium acetate, $20 \mathrm{mM}$ Tris-acetate, $10 \mathrm{mM}$ magnesium acetate, $1 \mathrm{mM}$ DTT, $\mathrm{pH}$ 7.9) were purchased from New England Biolabs. Unless otherwise noted, all chemicals were purchased from Sigma-Aldrich.

All DNA were purchased from Microsynth. The sequences each contain one specific cutting site for one of the type-II REs, either BamHI (GGATCC) or EcoRV (GATATC). In order to noncovalently anchor dsDNA on the SWCNT surface, three different configurations were designed, as shown in Figure 1a. Cy3-modified DNA was purified by PAGE by the manufacturer. Complementary DNA sequences were annealed in $\mathrm{ddH}_{2} \mathrm{O}$ by incubation at $95^{\circ} \mathrm{C}$ for 5 min followed by slow cooling to room temperature $(\sim 2 \mathrm{~h})$.
Preparation of dsDNA-SWCNT. We prepared ssDNAdsDNA-SWCNT hybrids according to the method described by Shiraki et al. with some modifications (summarized in the Supporting Information). In brief, the SC concentration of the SC-suspended SWCNT was adjusted by dialyzing against $0.5 \mathrm{mM} \mathrm{SC}$. The resulting SWCNT suspension was incubated with thermally annealed dsDNA in excess at room temperature for at least 3 days in order to reach equilibrium. Replaced SC and free DNA were removed with Amicon Ultra-2 $\mathrm{mL}$ centrifugal filters (Millipore, $\mathrm{MWCO}=100 \mathrm{kDa}$ ) washing 6 times with $1 \mathrm{~mL}$ of $\mathrm{ddH}_{2} \mathrm{O}$ (see the Supporting Information). The filtered solutions were collected and centrifuged at $21130 \mathrm{~g}$ for $10 \mathrm{~min}$ to remove unwanted SWCNT bundles. The dsDNA-SWCNT solutions were stored at $4{ }^{\circ} \mathrm{C}$, where they can remain stable for months. The concentration of SWCNTs was estimated from the absorption spectrum, taking $\varepsilon_{910 \mathrm{~nm}}$ of $0.02554 \mathrm{~L} \mathrm{mg}^{-1} \mathrm{~cm}^{-1} .{ }^{56}$ Details on photoluminescence and AFM characterization of hybrids can be found in the Supporting Information.

Digestion Assay. We developed an endonuclease assay to study conformations of dsDNA on SWCNTs. The scheme is shown in Figure $1 \mathrm{~b}$. In a typical experiment, $0.5 \mathrm{mg} / \mathrm{L}$ of DNA-SWCNTs was incubated with $20 \mathrm{U}$ of BamHI-HF or EcoRV-HF in NEBuffer $4(1 \times)$ in a total volume of $30 \mu \mathrm{L}$ in $1.5 \mathrm{~mL}$ Eppendorf tubes, at $22^{\circ} \mathrm{C}$ and shaking with $300 \mathrm{rpm}$. Aliquots supplemented with Triton X-100 were incubated with the desired Triton X-100 concentration for $2 \mathrm{~h}$ at 22 ${ }^{\circ} \mathrm{C}$ before mixing with the enzyme and buffer. After $16 \mathrm{~h}$ of incubation, the reaction solutions were diluted to $300 \mu \mathrm{L}$ with $\mathrm{ddH}_{2} \mathrm{O}$ before being stopped by adding an equal volume of phenolchloroform-isoamyl alcohol extraction reagent (Sigma-Aldrich, 25:24:1). After centrifugation for $5 \mathrm{~min}$ at $16000 \mathrm{~g}$ at room temperature, the aqueous phase was collected, and the DNA was precipitated according to literature using ethanol supplemented with glycogen (Carl Roth). ${ }^{57}$ The pellet was washed with $70 \%$ ethanol and resuspended in denaturing gel loading buffer containing 95\% formamide. Samples were heat-denatured for $3 \mathrm{~min}$ at $95{ }^{\circ} \mathrm{C}$ and immediately quenched on ice before separation on a denaturing $12 \%$ urea-polyacrylamide gel in $1 \times$ Tris-boric acid-EDTA buffer at $250 \mathrm{~V}$ for $45 \mathrm{~min}$. The DNA was stained with SYBR Gold (Thermo Fisher) for $\sim 25 \mathrm{~min}$ and visualized on a blue-light gel imager. The amount of DNA was quantified using ImageJ. All experiments were conducted in triplicate.

Fluorescence Measurements. Twenty microliter sample aliquots were placed in a flat-bottom 384-well plate (Nunc MaxiSorp). Visible-range emission spectra were recorded using a Varioskan microplate reader.

MD Simulation. For the initial condition, we conjugated the dsDNA segment of $\mathrm{BH} 7-5$ aligned along the nanotube axis to an equilibrium conformation of $(\mathrm{GT})_{10}$ on the $(9,4)$-SWCNT, which was modeled by Harvey et al. ${ }^{9}$ The surrounding aqueous solution contained $30 \mathrm{mM}$ sodium and $10 \mathrm{mM}$ magnesium ions. After $100 \mathrm{~ns}$ equilibration, the simulation was subsequently run for $250 \mathrm{~ns}$. The final equilibrated snapshot is shown in Figure 5a. Additional details on the simulation can be found in the Supporting Information

\section{ASSOCIATED CONTENT}

\section{Supporting Information}

The Supporting Information is available free of charge on the ACS Publications website at DOI: 10.1021/acsami.8b12287.

Additional experimental details, data, and analyses; DNA sequences used in the study; and MD simulation details (PDF)

Video of MD simulation results (AVI)

\section{AUTHOR INFORMATION}

\section{Corresponding Author}

*E-mail: ardemis.boghossian@epfl.ch.

ORCID

Shang-Jung Wu: 0000-0003-0097-1774 
Alice J. Gillen: 0000-0002-8328-1017

Clémence Corminboeuf: 0000-0001-7993-2879

Ardemis A. Boghossian: 0000-0001-6155-3985

\section{Notes}

The authors declare no competing financial interest.

\section{ACKNOWLEDGMENTS}

The authors thank Daniel Roxbury and Jeetain Mittal for providing the initial configuration file for the MD simulations. They are also thankful for funding support from the Swiss National Science Foundation (SNSF) Assistant Professor (AP) Energy Grant.

\section{REFERENCES}

(1) Seow, Y.; Wood, M. J. Biological Gene Delivery Vehicles: Beyond Viral Vectors. Mol. Ther. 2009, 17, 767-777.

(2) McNicol, A. M.; Farquharson, M. A. In Situ Hybridization and Its Diagnostic Applications in Pathology. J. Pathol. 1997, 182, 250261.

(3) Hong, F.; Zhang, F.; Liu, Y.; Yan, H. DNA Origami: Scaffolds for Creating Higher Order Structures. Chem. Rev. 2017, 117, 1258412640.

(4) Famulok, M.; Mayer, G. Aptamer Modules as Sensors and Detectors. Acc. Chem. Res. 2011, 44, 1349-1358.

(5) Cunningham, F. J.; Goh, N. S.; Demirer, G. S.; Matos, J. L.; Landry, M. P. Nanoparticle-Mediated Delivery Towards Advancing Plant Genetic Engineering. Trends Biotechnol. 2018, 36, 882-897.

(6) Mout, R.; Ray, M.; Yesilbag Tonga, G.; Lee, Y.-W.; Tay, T.; Sasaki, K.; Rotello, V. M. Direct Cytosolic Delivery of CRISPR/Cas9Ribonucleoprotein for Efficient Gene Editing. ACS Nano 2017, 11, $2452-2458$

(7) Godin, A. G.; Varela, J. A.; Gao, Z.; Danné, N.; Dupuis, J. P.; Lounis, B.; Groc, L.; Cognet, L. Single-Nanotube Tracking Reveals the Nanoscale Organization of the Extracellular Space in the Live Brain. Nat. Nanotechnol. 2017, 12, 238-243.

(8) Jena, P. V.; Roxbury, D.; Galassi, T. V.; Akkari, L.; Horoszko, C. P.; Iaea, D. B.; Budhathoki-Uprety, J.; Pipalia, N.; Haka, A. S.; Harvey, J. D.; Mittal, J.; Maxfield, F. R.; Joyce, J. A.; Heller, D. A. A Carbon Nanotube Optical Reporter Maps Endolysosomal Lipid Flux. ACS Nano 2017, 11, 10689-10703.

(9) Harvey, J. D.; Jena, P. V.; Baker, H. A.; Zerze, G. H.; Williams, R. M.; Galassi, T. V.; Roxbury, D.; Mittal, J.; Heller, D. A. A Carbon Nanotube Reporter of Microrna Hybridization Events in Vivo. Nat. Biomed. Eng. 2017, 1, 0041.

(10) Wong, M. H.; Misra, R. P.; Giraldo, J. P.; Kwak, S.-Y.; Son, Y.; Landry, M. P.; Swan, J. W.; Blankschtein, D.; Strano, M. S. Lipid Exchange Envelope Penetration (LEEP) of Nanoparticles for Plant Engineering: A Universal Localization Mechanism. Nano Lett. 2016, 16, 1161-1172.

(11) Boyer, P. D.; Ganesh, S.; Qin, Z.; Holt, B. D.; Buehler, M. J.; Islam, M. F.; Dahl, K. N. Delivering Single-Walled Carbon Nanotubes to the Nucleus Using Engineered Nuclear Protein Domains. ACS Appl. Mater. Interfaces 2016, 8, 3524-3534.

(12) Zheng, M.; Jagota, A.; Semke, E. D.; Diner, B. A.; Mclean, R. S.; Lustig, S. R.; Richardson, R. E.; Tassi, N. G. DNA-Assisted Dispersion and Separation of Carbon Nanotubes. Nat. Mater. 2003, 2, 338-342.

(13) Tu, X.; Manohar, S.; Jagota, A.; Zheng, M. DNA Sequence Motifs for Structure-Specific Recognition and Separation of Carbon Nanotubes. Nature 2009, 460, 250-253.

(14) Kupis-Rozmysłowicz, J.; Antonucci, A.; Boghossian, A. A. Review-Engineering the Selectivity of the DNA-SWCNT Sensor. ECS J. Solid State Sci. Technol. 2016, 5, M3067-M3074.

(15) Jena, P. V.; Safaee, M. M.; Heller, D. A.; Roxbury, D. DNACarbon Nanotube Complexation Affinity and Photoluminescence Modulation Are Independent. ACS Appl. Mater. Interfaces 2017, 9, 21397-21405.
(16) Ao, G.; Streit, J. K.; Fagan, J. A.; Zheng, M. Differentiating Leftand Right-Handed Carbon Nanotubes by DNA. J. Am. Chem. Soc. 2016, 138, 16677-16685.

(17) Wu, Y.; Phillips, J. A.; Liu, H.; Yang, R.; Tan, W. Carbon Nanotubes Protect DNA Strands During Cellular Delivery. ACS Nano 2008, 2, 2023-2028.

(18) Ghosh, S.; Patel, N.; Chakrabarti, R. Probing the Salt Concentration Dependent Nucleobase Distribution in a SingleStranded DNA-Single-Walled Carbon Nanotube Hybrid with Molecular Dynamics. J. Phys. Chem. B 2016, 120, 455-466.

(19) Han, S.-p.; Maune, H. T.; Barish, R. D.; Bockrath, M.; Goddard, W. A. DNA-Linker-Induced Surface Assembly of Ultra Dense Parallel Single Walled Carbon Nanotube Arrays. Nano Lett. 2012, 12, 1129-1135.

(20) Wang, C.; Meany, B.; Wang, Y. Optically Triggered Melting of DNA on Individual Semiconducting Carbon Nanotubes. Angew. Chem., Int. Ed. 2017, 56, 9326-9330.

(21) Müller, K.; Malik, S.; Richert, C. Sequence-Specifically Addressable Hairpin DNA-Single-Walled Carbon Nanotube Complexes for Nanoconstruction. ACS Nano 2010, 4, 649-656.

(22) Zhao, X.; Johnson, J. K. Simulation of Adsorption of DNA on Carbon Nanotubes. J. Am. Chem. Soc. 2007, 129, 10438-10445.

(23) Yamamoto, Y.; Fujigaya, T.; Niidome, Y.; Nakashima, N. Fundamental Properties of Oligo Double-Stranded DNA/SingleWalled Carbon Nanotube Nanobiohybrids. Nanoscale 2010, 2, 17671772.

(24) Ao, G.; Nepal, D.; Aono, M.; Davis, V. A. Cholesteric and Nematic Liquid Crystalline Phase Behavior of Double-Stranded DNA Stabilized Single-Walled Carbon Nanotube Dispersions. ACS Nano 2011, 5, 1450-1458.

(25) Nii, D.; Hayashida, T.; Yamaguchi, Y.; Ikawa, S.; Shibata, T.; Umemura, K. Selective Binding of Single-Stranded DNA-Binding Proteins onto DNA Molecules Adsorbed on Single-Walled Carbon Nanotubes. Colloids Surf., B 2014, 121, 325-330.

(26) Cathcart, H.; Nicolosi, V.; Hughes, J. M.; Blau, W. J.; Kelly, J. M.; Quinn, S. J.; Coleman, J. N. Ordered DNA Wrapping Switches on Luminescence in Single-Walled Nanotube Dispersions. J. Am. Chem. Soc. 2008, 130, 12734-12744.

(27) Chen, R. J.; Zhang, Y. Controlled Precipitation of Solubilized Carbon Nanotubes by Delamination of DNA. J. Phys. Chem. B 2006, $110,54-57$.

(28) Jung, S.; Cha, M.; Park, J.; Jeong, N.; Kim, G.; Park, C.; Ihm, J.; Lee, J. Dissociation of Single-Strand DNA: Single-Walled Carbon Nanotube Hybrids by Watson-Crick Base-Pairing. J. Am. Chem. Soc. 2010, 132, 10964-10966.

(29) Jeng, E. S.; Barone, P. W.; Nelson, J. D.; Strano, M. S. Hybridization Kinetics and Thermodynamics of DNA Adsorbed to Individually Dispersed Single-Walled Carbon Nanotubes. Small 2007, 3, 1602-1609.

(30) Pingoud, A.; Fuxreiter, M.; Pingoud, V.; Wende, W. Type II Restriction Endonucleases: Structure and Mechanism. Cell. Mol. Life Sci. 2005, 62, 685-707.

(31) Azorin, F.; Hahn, R.; Rich, A. Restriction Endonucleases Can Be Used to Study B-Z Junctions in Supercoiled DNA. Proc. Natl. Acad. Sci. U.S.A. 1984, 81, 5714-5718.

(32) Shouche, Y. S.; Ramesh, N.; Brahachari, S. K. Probing of Unusual DNA Structures in Topologically Constrained Form V DNA: Use of Restriction Enzymes as Structural Probe. Nucleic Acids Res. 1990, 18, 267-275.

(33) Shiraki, T.; Tsuzuki, A.; Toshimitsu, F.; Nakashima, N. Thermodynamics for the Formation of Double-Stranded DNA-SingleWalled Carbon Nanotube Hybrids. Chem.-Eur. J. 2016, 22, 47744779.

(34) Bergler, F. F.; Stahl, S.; Goy, A.; Schöppler, F.; Hertel, T. Substrate-Mediated Cooperative Adsorption of Sodium Cholate on (6,5) Single-Wall Carbon Nanotubes. Langmuir 2016, 32, 95989603. 
(35) Conlan, L. H.; José, T. J.; Thornton, K. C.; Dupureur, C. M. Modulating Restriction Endonuclease Activities and Specificities Using Neutral Detergents. BioTechniques 1999, 27, 955-960.

(36) Kruss, S.; Landry, M. P.; Vander Ende, E.; Lima, B. M. A.; Reuel, N. F.; Zhang, J.; Nelson, J.; Mu, B.; Hilmer, A.; Strano, M. Neurotransmitter Detection Using Corona Phase Molecular Recognition on Fluorescent Single-Walled Carbon Nanotube Sensors. J. Am. Chem. Soc. 2014, 136, 713-724.

(37) Muddana, H. S.; Morgan, T. T.; Adair, J. H.; Butler, P. J. Photophysics of Cy3-Encapsulated Calcium Phosphate Nanoparticles. Nano Lett. 2009, 9, 1559-1566.

(38) Chhabra, R.; Sharma, J.; Wang, H.; Zou, S.; Lin, S.; Yan, H.; Lindsay, S.; Liu, Y. Distance-Dependent Interactions between Gold Nanoparticles and Fluorescent Molecules with DNA as Tunable Spacers. Nanotechnology 2009, 20, 485201.

(39) Landry, M. P.; Ando, H.; Chen, A. Y.; Cao, J.; Kottadiel, V. I.; Chio, L.; Yang, D.; Dong, J.; Lu, T. K.; Strano, M. S. Single-Molecule Detection of Protein Efflux from Microorganisms Using Fluorescent Single-Walled Carbon Nanotube Sensor Arrays. Nat. Nanotechnol. 2017, 12, 368-377.

(40) Jen-Jacobson, L. Protein-DNA recognition complexes: Conservation of structure and binding energy in the transition state. Biopolymers 1997, 44, 153-180.

(41) Krissinel, E.; Henrick, K. Inference of Macromolecular Assemblies from Crystalline State. J. Mol. Biol. 2007, 372, 774-797.

(42) Kostrewa, D.; Winkler, F. K. $\mathrm{Mg}^{2+}$ Binding to the Active Site of EcoRV Endonuclease: A Crystallographic Study of Complexes with Substrate and Product DNA at 2 A Resolution. Biochemistry 1995, 34, 683-696.

(43) Winkler, F. K.; Banner, D. W.; Oefner, C.; Tsernoglou, D.; Brown, R. S.; Heathman, S. P.; Bryan, R. K.; Martin, P. D.; Petratos, K.; Wilson, K. S. The Crystal Structure of EcoRV Endonuclease and of Its Complexes with Cognate and Non-Cognate DNA Fragments. EMBO J. 1993, 12, 1781-1795.

(44) van den Broek, B.; Noom, M. C.; Wuite, G. J. L. DNA-Tension Dependence of Restriction Enzyme Activity Reveals Mechanochemical Properties of the Reaction Pathway. Nucleic Acids Res. 2005, 33, 2676-2684.

(45) Viadiu, H.; Aggarwal, A. K. Structure of BamHI Bound to Nonspecific DNA. Mol. Cell 2000, 5, 889-895.

(46) Ghosh, S.; Chakrabarti, R. Spontaneous Unzipping of Xylonucleic Acid Assisted by a Single-Walled Carbon Nanotube: A Computational Study. J. Phys. Chem. B 2016, 120, 3642-3652.

(47) Alegret, N.; Santos, E.; Rodríguez-Fortea, A.; Rius, F. X.; Poblet, J. M. Disruption of Small Double Stranded DNA Molecules on Carbon Nanotubes: A Molecular Dynamics Study. Chem. Phys. Lett. 2012, 525-526, 120-124.

(48) Santosh, M.; Panigrahi, S.; Bhattacharyya, D.; Sood, A. K.; Maiti, P. K. Unzipping and Binding of Small Interfering RNA with Single Walled Carbon Nanotube: A Platform for Small Interfering RNA Delivery. J. Chem. Phys. 2012, 136, 065106.

(49) SantaLucia, J.; Allawi, H. T.; Seneviratne, P. A. Improved Nearest-Neighbor Parameters for Predicting DNA Duplex Stability†. Biochemistry 1996, 35, 3555-3562.

(50) Jeltsch, A.; Alves, J.; Wolfes, H.; Maass, G.; Pingoud, A. Pausing of the Restriction Endonuclease EcoRI During Linear Diffusion on DNA. Biochemistry 1994, 33, 10215-10219.

(51) Yi, C.; Fong, C.-C.; Chen, W.; Qi, S.; Tzang, C.-H.; Lee, S.-T.; Yang, M. Interactions between Carbon Nanotubes and DNA Polymerase and Restriction Endonucleases. Nanotechnology 2007, 18,025102 .

(52) Ordinario, D. D.; Burke, A. M.; Phan, L.; Jocson, J.-M.; Wang, H.; Dickson, M. N.; Gorodetsky, A. A. Sequence Specific Detection of Restriction Enzymes at DNA-Modified Carbon Nanotube Field Effect Transistors. Anal. Chem. 2014, 86, 8628-8633.

(53) Olsen, T. J.; Choi, Y.; Sims, P. C.; Gul, O. T.; Corso, B. L.; Dong, C.; Brown, W. A.; Collins, P. G.; Weiss, G. A. Electronic Measurements of Single-Molecule Processing by DNA Polymerase I (Klenow Fragment). J. Am. Chem. Soc. 2013, 135, 7855-7860.
(54) van de Ven, A. L.; Adler-Storthz, K.; Richards-Kortum, R. Delivery of Optical Contrast Agents Using Triton-X100, Part 1: Reversible Permeabilization of Live Cells for Intracellular Labeling. J. Biomed. Optic. 2009, 14, 021012.

(55) Li, B.; Wang, L.; Su, L.; Chen, S.; Li, Z.; Chen, J.; Wu, J. Glycine and Triton X-100 Enhanced Secretion of Recombinant $\alpha$ CGTase Mediated by OmpA Signal Peptide in Escherichia Coli. Biotechnol. Bioprocess Eng. 2012, 17, 1128-1134.

(56) Roxbury, D.; Jena, P. V.; Williams, R. M.; Enyedi, B.; Niethammer, P.; Marcet, S.; Verhaegen, M.; Blais-Ouellette, S.; Heller, D. A. Hyperspectral Microscopy of near-Infrared Fluorescence Enables 17-Chirality Carbon Nanotube Imaging. Sci. Rep. 2015, 5, 14167.

(57) Green, M. R. Molecular Cloning: A Laboratory Manual; Green, M. R., Sambrook, J., Ed.; Cold Spring Harbor Laboratory Press: Cold Spring Harbor, N.Y, 2012. 